\title{
Inhibitors of poly ADP-ribose polymerase (PARP) induce apoptosis of myeloid leukemic cells: potential for therapy of myeloid leukemia and myelodysplastic syndromes
}

\section{Terry J Gaymes, Sydney Shall, Lee J Macpherson, Natalie A Twine, Nicholas C Lea, Farzin Farzaneh and Ghulam J Mufti}

Department of Haematological Medicine, King's College London, Leukemia Sciences Laboratories, The Rayne Institute, Denmark Hill campus, London, UK

In the original article, there was a mistake in Figure 3D as published. In this instance, the annexin $\mathrm{V}$ fluorescence-activated cell sorting (FACS) image for Figure 1B (top right) had been unintentionally duplicated and substituted for the annexin V FACS image in Figure 3D (top left). As it is an untreated drug control rather than drug test additions, no scientific benefit or advantage interpretation could be made by duplicating the data here. The authors apologize for this error and state that this does not change the scientific conclusions of the article in any way. The amended Figure 3 is attached.

An incorrect version of Figure 3 appared in the May 2009 Issue on page 644. The correct version of Figure 3 is published on this page.

A

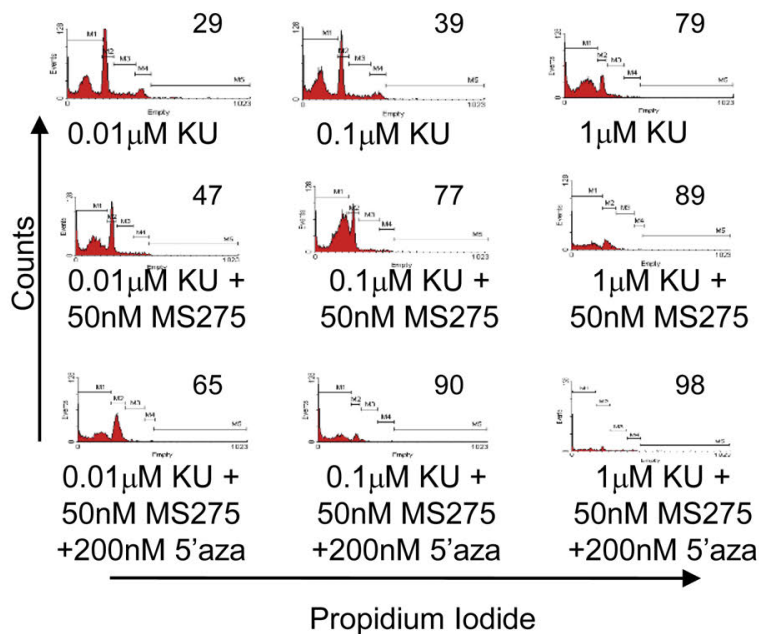

C

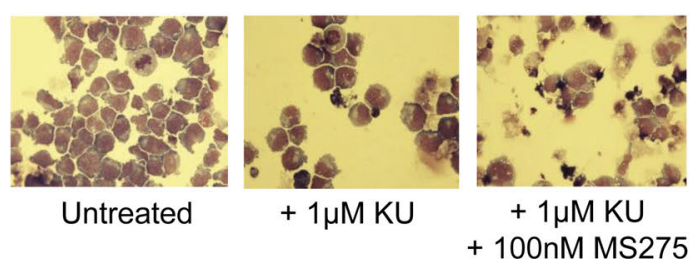

B
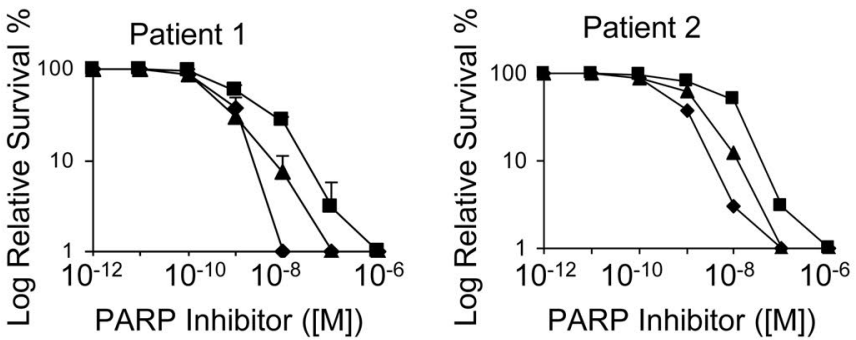

D

P39

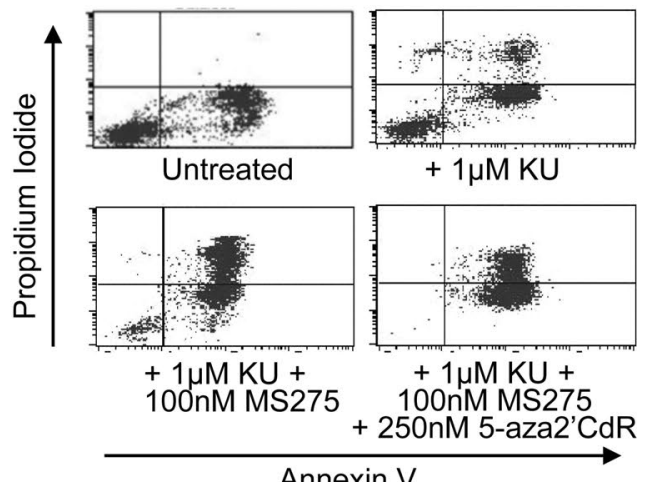

Figure 3. The effect on leukemic cells of the PARP inhibitor, KU in combination with non-cytotoxic concentrations of 5'-aza-2'-dCR and/or HDAC inhibitor. (A) The effect on primary acute myeloid leukemia (AML) cells of the PARP inhibitor, KU in combination with the HDAC inhibitor, MS275 and with 5'-aza-2'-dCR. KU was added at variable concentrations to Patient 2 continuously for 5 days with 50 nM MS275 and 200 nM 5'-aza-2'-dCR or KU alone and then analyzed by flow cytometry. The apoptotic index (Sub-G1population as a fraction of sub-G1+ G1 populations is shown in the right inset). (B) Trypan blue exclusion assays were used to determine cell survival in primary AML cells of Patient 2 exposed to KU, MS275 and 5'-aza-2'-dCR. Exposure to 50 nM MS275 and 200 nM 5'-aza-2'-dCR with varying concentrations of KU was continuous for 5 days in AML cells of Patient 1 and AML cells of Patient 2 cells. KU + MS275 (triangles), 5'-aza-2'-dCR + KU + MS275 (diamonds). KU alone (squares). (C) P39 cells were cultured in 1 uM KU, 1 uM KU + 100 nM MS275 or left untreated for 7 days. At 7 days cells were cytospun onto slides and stained with May-Grumwald stain. Images were taken at $40 \mathrm{X}$ magnification. (D) $1 \mu \mathrm{M}$ KU, $100 \mathrm{nM}$ MS275 and 250 nM 5'-aza-2'-CdR were added as indicated to P39 for 7 days before being treated with annexin V-FITC (FL-1, X-axis) and propidium iodide (FL-2, Y-axis). The figures show the percentage of early and late apoptotic cells in the population. 